ROSEU MiEKO YAMAMOTO NOMURA ${ }^{1}$

Marina VIIELA Chagas Ferreira ${ }^{2}$ IBRAHIM OMAR ABDel Latif ${ }^{2}$

Rossana Pulaneu Viera Franaisco' MARCEO ZUGABB

\section{Artigo Origina}

Palavras-chave

Movimento fetal

Gravidez

Feto

Ultrassonografia pré-natal

Keywords

Fetal movements Pregnancy

Fetus

Ultrasonography, prenatal

\title{
Concordância entre a percepção materna dos movimentos fetais e a visualização pela ultrassonografia
}

\author{
Agreement between maternal perception of fetal movements and \\ visualization by ultrasound
}

\section{Resumo}

OBJETIVO: Verificar, em gestantes de baixo risco, a concordância entre a percepção materna dos movimentos fetais e os movimentos constatados simultaneamente pela ultrassonografia. MÉTODOS: Foram avaliadas 20 gestantes com os seguintes critérios de inclusão: feto único e vivo; idade materna entre 18 e 35 anos; idade gestacional entre 36 e 40 semanas completas; morfologia fetal normal ao exame ultrassonográfico; ausência de morbidades clínicas ou obstétricas. A gestante foi avaliada por dez minutos, período em que foi monitorada com aparelho de cardiotocografia para registro dos movimentos fetais acionados com o marcador de eventos, com a velocidade do papel ajustada para $3 \mathrm{~cm} / \mathrm{min}$. Simultaneamente foi realizada observação dos movimentos fetais pela ultrassonografia. RESULTADOS: A análise de concordância interobservador pela análise de kappa obteve o índice de 0,62, caracterizando boa concordância (IC95\% 0,45-0,79). O coeficiente de correlação intraclasse foi de 0,82 (IC95\% 0,61-0,92). A análise pelo gráfico de Bland \& Altman indicou boa concordância. A análise pela regressão linear demonstrou correlação significativa entre a percepção materna $(x)$ e a observação pela ultrassonografia $(y)\left(r^{2}=0,71 ; p<0,001\right.$; equação: $y=5,31+0,66 x)$. CONCLUSÕES: A concordância da percepção materna e ultrassonográfica dos movimentos fetais é boa, o que permite o uso da contagem de movimentos fetais na avaliação da vitalidade fetal.

\section{Abstract}

PURPOSE: To determine the agreement between maternal perception of fetal movements and the movements recorded simultaneously by ultrasound in low-risk pregnancies. METHODS: Twenty pregnant women were evaluated with the following inclusion criteria: single pregnancy, alive fetus, maternal age between 18 and 35 years; between 36 and 40 weeks gestation; normal fetal morphology at ultrasound, and absence of maternal comorbidities. The pregnant women were evaluated for 10 minutes, during which cardiotocography was used to record fetal movements triggered with the event marker, with the paper speed set at $3 \mathrm{~cm} / \mathrm{min}$. At the same time, fetal movements were observed by ultrasonography. RESULTS: The kappa index for interobserver agreement analysis was 0.62 , showing good agreement (95\% Cl 0.45-0.79). The intraclass correlation coefficient was $0.82(95 \% \mathrm{Cl} 0.61-0.92)$. Analysis by the Bland \& Altman graph indicated good agreement. A linear regression analysis showed a significant correlation between maternal perception $(x)$ and ultrasound $(y)$ observation $\left(r^{2}=0.71, p<0.001\right.$; equation: $\left.y=5.31+0.66 x\right)$. CONCLUSIONS: The agreement between ultrasound and maternal perception of fetal movement is good, allowing the use of fetal movement counting in the assessment of fetal wellbeing.

Correspondêncio

Roseli Mieko Yamamoto Nomuro Departamento de Obsteticica e Ginecologia, Foculdade de Medicina da Universidade de São Paulo Avenida Dr. Enéas de Carvalho Aguiar, $255,10^{\circ}$ andar, sala 10037 (EP: 05403-000

São Paulo (SP), Brasil

Recebido

$23 / 10 / 2012$

Aceito com modificacōes $26 / 11 / 2012$
Trabalho realizado na disciplina de Obstetrícia, Departamento de Obstetrícia e Ginecologia da Faculdade de Medicina, Universidade de São Paulo - USP - São Paulo (SP), Brasil.

'Disciplina de Obstetrícia, Departamento de Obstetrícia e Ginecologia da Faculdade de Medicina, Universidade de São Paulo USP - São Paulo (SP), Brasil.

${ }^{2}$ Graduação em Medicina da Faculdade de Medicina, Universidade de São Paulo - USP - São Paulo (SP) - Brasil.

${ }^{3}$ Departamento de Obstetrícia e Ginecologia da Faculdade de Medicina, Universidade de São Paulo - USP - São Paulo (SP), Brasil. 


\section{Introdução}

Os movimentos fetais (MF) são de grande importância para a vigilância do bem-estar fetal ${ }^{1}$ e constituem um dos métodos mais antigos utilizado na sinalização da condição fetal no período anteparto ${ }^{2}$. A redução da movimentação fetal associa-se a maior risco de prematuridade, baixo peso do recém-nascido, ou mesmo de morte fetal no período anteparto ${ }^{3-5}$. Portanto, a queixa de diminuição da movimentação fetal é um dos primeiros sinais clínicos de comprometimento da vitalidade do produto conceptual.

A percepção materna dos MF inicia-se por volta da $16^{\mathrm{a}}-20^{\mathrm{a}}$ semana, mas a frequência de MF não é constante durante toda a gravidez. A gestante pode mensurar regularmente os MF, o que seria uma importante ferramenta para avaliar a vitalidade fetal e detectar precocemente qualquer problema que necessite de intervenção. Kuwata et al. ${ }^{6}$ relatam estudo sobre o intervalo de tempo que 705 pacientes levam para contabilizar $10 \mathrm{MF}$ (método "count to 10 modificado"), e verificam que isso ocorre em $10 \mathrm{a}$ 15 minutos.

Muitos estudos abordam a redução da mortalidade fetal quando são aplicados programas que orientam as gestantes sobre a importância da vigilância dos $\mathrm{MF}^{7}$. Entretanto, há controvérsias na literatura acerca de sua aplicabilidade. Os estudos são conflitantes e alguns não demonstram benefícios quando são aplicados protocolos de contagem de $\mathrm{MF}^{8}$. Frøen et al. ${ }^{9}$ ressaltam que apesar de a percepção de MF estar associada com melhores resultados perinatais, a busca de um "limite de alarme", para definir diminuição dos MF, tem sido até agora mal-sucedida. Durante a contagem de movimentos fetais pode ocorrer grande número de falsos-positivos ou falsos-negativos ${ }^{10,11}$. Nesse sentido, o conhecimento da confidencialidade da percepção materna dos MF torna-se relevante. O presente estudo, realizado em gestantes de baixo risco, sem morbidade, teve como objetivo verificar a concordância entre a percepção materna dos MF e o movimento constatado simultaneamente pela ultrassonografia.

\section{Métodos}

Este trabalho foi realizado em hospital universitário e a população incluída foi selecionada entre gestantes de baixo risco, acompanhadas no Ambulatório da Liga de Pré-natal da Clínica Obstétrica do Hospital das Clínicas da Faculdade de Medicina da Universidade de São Paulo, no período compreendido entre abril de 2011 e agosto de 2012. Foram convidadas 20 gestantes e todas consentiram em participar da pesquisa. O estudo foi do tipo prospectivo e transversal. $\mathrm{O}$ projeto de pesquisa e o termo de consentimento livre e esclarecido foram aprovados pela Comissão de Ética em Pesquisa do Hospital das Clínicas da Faculdade de Medicina da Universidade de São Paulo - CAPPesq, sob o número 193/11.

Foram utilizados os seguintes critérios de inclusão: idade materna entre 18 e 35 anos, gestação única, feto vivo, com idade gestacional entre 36 e 40 semanas completas, ausência de intercorrências clínicas ou obstétricas, ultrassonografia morfológica fetal normal e concordância formal da paciente em participar do estudo. Foi utilizado como critério de exclusão o diagnóstico pós-natal de anomalia fetal. Todas as pacientes incluídas foram submetidas à avaliação sob as mesmas condições. Foram avaliadas na sala de exames de vitalidade fetal, no horário entre $12 \mathrm{~h} 00 \mathrm{e}$ $15 \mathrm{~h} 00$, em posição semissentada e após consulta de rotina no pré-natal. A idade gestacional foi calculada a partir da data da última menstruação (DUM), quando compatível com a idade gestacional estimada pela ultrassonografia realizada, no máximo, até a vigésima semana de gestação. Nos casos em que não foi observada tal concordância, a idade gestacional foi calculada pelos dados da primeira ultrassonografia (USG).

A percepção materna dos MF foi avaliada utilizando-se o aparelho de cardiotocografia (marca Philips HP50A). Os transdutores de detecção dos batimentos cardíacos fetais e das contrações uterinas não foram utilizados. Foi empregado apenas o marcador de eventos, que, quando acionado, registra no papel cardiotocográfico uma marca específica. Esse dispositivo foi utilizado para que a gestante pudesse indicar objetivamente o MF percebido, sem que o operador do aparelho de USG tivesse conhecimento. A velocidade do papel de registro da cardiotocografia foi ajustada para $3 \mathrm{~cm} / \mathrm{min}$. A observação ultrassonográfica dos MF foi realizada com aparelho da marca Toshiba (modelo Corevision), com transdutor de $3,5 \mathrm{MHz}$, posicionado na parede abdominal materna de forma a incluir o tronco fetal e pelo menos um dos membros. Durante o período de 10 minutos foi solicitado que a gestante registrasse os MF percebidos, e, simultaneamente, foi realizada a observação pela USG. O horário do início do período de observação foi marcado no papel da cardiotocografia e pelo registro do horário em uma primeira fotografia impressa de USG. A cada MF percebido, a gestante foi instruída a acionar o botão "marcador de eventos". Posteriormente, essas marcações foram confrontadas com a observação ultrassonog ráfica dos MF, que foram registrados pelo operador do equipamento de ultrassom na forma de fotografias impressas. Todos os exames foram realizados exclusivamente por uma pesquisadora (M.V.C.F.), sob supervisão de R.M.Y.N.

Foram comparados os movimentos observados pela gestante e os detectados pelo operador do aparelho de 
ultrassonografia, cotejando o horário em que ocorreram. Comparou-se o número total de MF indicados pela gestante com os observados com a USG, bem como a concordância da observação dos MF (foram considerados concordantes movimentos indicados com diferença de até 20 segundos).

Foram analisados os exames de 20 gestantes. $\mathrm{Na}$ Tabela 1 estão apresentadas as informações referentes à caracterização dessas pacientes. Os resultados foram analisados utilizando-se o programa Medcalc versão 11.5.1.0 (Medcalc Software, Bélgica). As variáveis foram analisadas descritivamente, calculando-se frequências absolutas e relativas. A concordância da percepção materna e ultrassonográfica de MF foi avaliada pela análise de concordância interobservador kappa de Cohen, que mede o grau de concordância além do que seria esperado tão somente pelo acaso, e varia entre 0 e 1 . Os valores próximos a 0 significam que a medida é atribuível apenas ao acaso, e valores próximos a 1 sugerem maior confiabilidade. A interpretação do grau de concordância da estatística kappa foi feita da seguinte maneira: nenhuma concordância, abaixo de zero; pobre, de 0 a 0,20; fraca, 0,21 a 0,40 ; satisfatória, de 0,41 a 0,60; boa, de 0,61 a 0,80 ; muito boa, de 0,81 a 0,92 , e excelente, de 0,93 a $1,00^{12}$. Foi utilizado também como método de comparação o gráfico de Bland \& Altman, usado para mensuração de variáveis clínicas e que permite a avaliação do padrão de concordância ou discordância entre medidas repetidas ${ }^{12}$. O nível de significância utilizado para os testes foi de $\mathrm{p}<0,05$.

\section{Resultados}

Os resultados do número de MF percebidos pela gestante e os observados na USG estão apresentados na Tabela 2. Nota-se que nem todos os movimentos são

Tabela 1. Características epidemiológicas e obstétricas das gestações em que foi realizada análise da percepcão materna dos movimentos fetais em período de 10 minutos $(n=20)$

\begin{tabular}{l|r}
\hline Parâmetro & Resultado \\
\hline $\begin{array}{l}\text { Idade materna, anos, média (DP) } \\
\text { Cor, n (\%) }\end{array}$ & $27,4(4,7)$ \\
$\quad$ Branca & $15(75,0)$ \\
$\quad$ Não branca & $5(25,0)$ \\
Paridade, n (\%) & \\
$\quad$ Nulípara & $4(20,0)$ \\
$\geq 1$ & $16(80,0)$ \\
Escolaridade, n (\%) & $8(40,0)$ \\
$\quad$ Ensino Fundamental & $10(50,0)$ \\
$\quad$ Ensino Médio & $2(10,0)$ \\
$\quad$ Ensino Superior & $373 / 7(36-385 / 7)$ \\
\hline Idade gestacional no exame, semanas, mediana \\
(min-máx)
\end{tabular}

detectados simultaneamente. A análise de concordância interobservador pela análise de kappa obteve o índice de 0,62 , caracterizando boa concordância, com desvio padrão de 0,08 e IC95\% 0,45-0,79.

O coeficiente de correlação intraclasse entre a percepção de movimentos fetais da gestante e a percepção ultrassonográfica foi de 0,82 (IC95\% 0,61-0,92). O resultado da análise pelo método de Bland \& Altman está apresentado na Figura 1. No eixo das abscissas (x) encontra-se a média dos MF percebidos pela gestante e pela USG, e no eixo das ordenadas (y) exibe-se a diferença entre os movimentos percebidos pela USG e pela gestante. A disposição dos pontos nos valores próximos a zero (0) indica uma boa concordância entre as percepções materna e ultrassonográfica dos MF, sem subestimação ou superestimação de nenhuma delas.

A análise de concordância pela regressão demonstrou correlação significativa entre a percepção materna e a observação pela USG. A Figura 2 demonstra a regressão linear do número de movimentos fetais detectados pela percepção materna (x) e pela USG (y), em período de 10 minutos de observação $\left(r^{2}=0,71 ; p<0,001\right.$; equação: $y=5,31+0,66 x)$.

Tabela 2. Percepção materna e ultrassonográfica dos movimentos fetais em período de 10 minutos de observaç̃̃o $(n=20)$

\begin{tabular}{lcccc}
\hline Características & Média & DP & Mediana & Min-máx \\
\hline MF percebidos pela mãe & 11,9 & 11,2 & 9 & $0-38$ \\
MF verificados na USG & 13,2 & 8,8 & 9,5 & $1-32$ \\
$\begin{array}{l}\text { MF defectados simultaneamente } \\
\text { (mãe e USG) }\end{array}$ & 8,3 & 7,0 & 6,5 & $1-25$ \\
\hline
\end{tabular}

MF: movimentos fetais; USG: Ultrassonografia; DP: desvio padrão; min: mínimo; máx: máximo

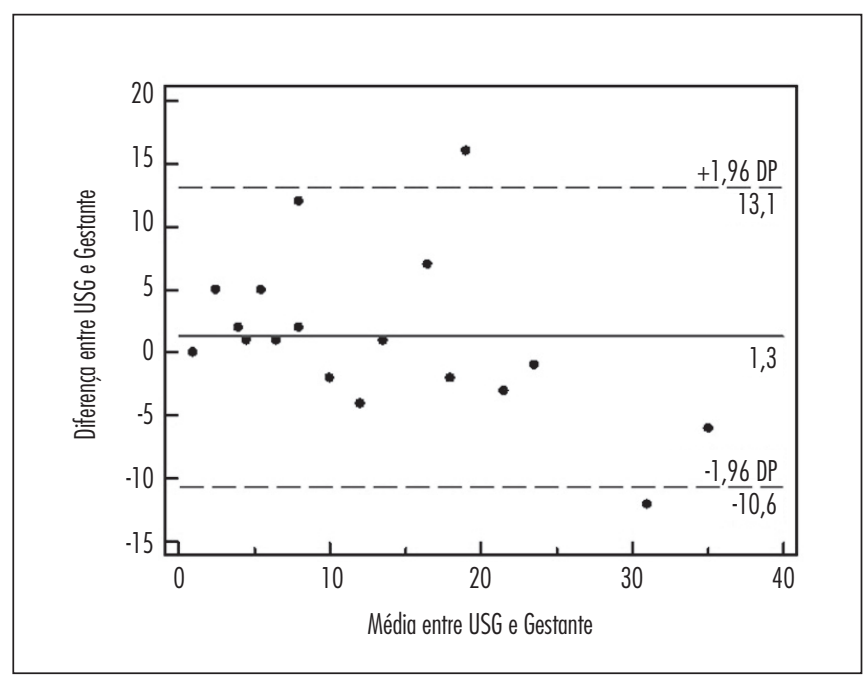

Figura 1. Gráfico de Bland \& Altman para a percepção materna e ultrassonográfica dos movimentos fetais $(n=20)$. Na abscissa $(x)$ tem-se a média e na ordenada (y) a diferença entre a percepção dos movimentos fetais pela ultrassonografia e pela gestante 


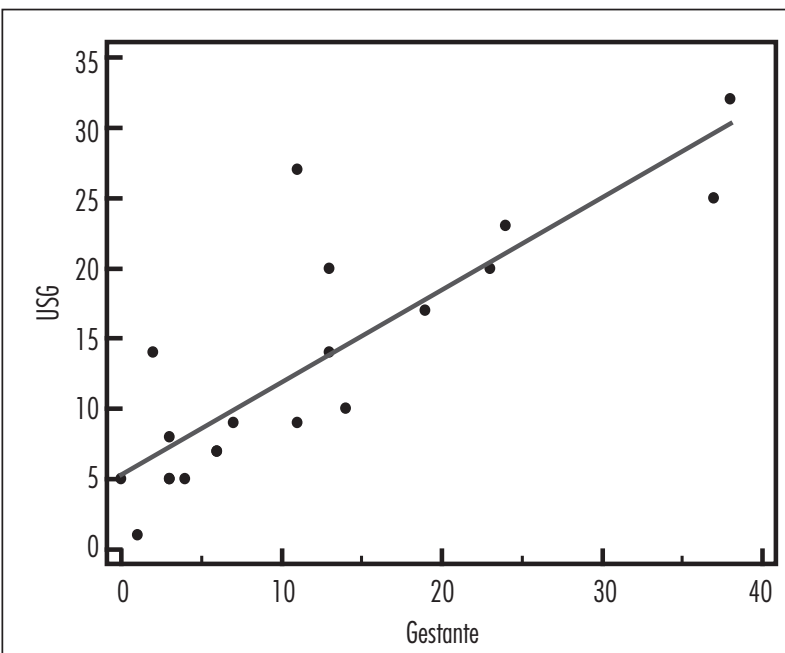

Figura 2. Gráfico de regressão linear do número de movimentos fetais detectados pela percepção materna $(x)$ e pela ultrassonografia $(y)$, em período de 10 minutos de observação $\left(r^{2}=0,71 ; p<0,001\right.$; equação: $y=5,31+0,66 x)$

\section{Discussão}

Com os resultados obtidos, o presente estudo sugere que é boa a concordância da percepção materna e ultrassonográfica dos movimentos fetais. Isso dá suporte para o uso do método de contagem materna dos movimentos fetais também em gestantes de baixo risco para avaliação contínua de bem-estar fetal em ambiente domiciliar. A confiabilidade da informação materna é aspecto relevante no cuidado pré-natal e a percepção materna dos movimentos fetais tem sido reconhecida como importante componente da assistência à saúde materno-fetal ${ }^{13}$.

Grande parte dos estudos envolvendo os MF e vitalidade fetal foi efetuada nas décadas de 1970 e 1980, época em que a contagem pela gestante foi muito estudada por ser técnica simples, de baixo custo e com grande aplicabilidade. Em revisão da literatura, foram analisados 24 estudos sobre a contagem de MF e verificou-se que a maioria deles foi realizada até a década de $1980^{14}$. A partir dessa época, com o desenvolvimento de novas tecnologias para avaliação fetal, tais como a cardiotocografia e perfil biofísico fetal, houve redução do interesse pelo tema.

Em estudo randomizado publicado em $1989^{8}$, foram avaliados 68.654 grávidas e não se demonstrou diferença significativa na prevenção de mortes fetais tardias, potencialmente evitáveis, entre gestantes instruídas a realizar contagem rotineira dos MF em relação ao Grupo Controle. No entanto, nota-se que a média do tempo relatado para contagem de $10 \mathrm{MF}$ foi de 162 minutos, o que difere do tempo relatado em estudo mais recente ( 10 a 15 minutos $)^{6}$. Além disso, o protocolo adotado para as mulheres com MF reduzidos não foi bem padronizado, havendo certa intersecção entre os grupos controle e experimental e não houve coleta de dados de morbidade perinatal.

Desde a década de 1970, autores relacionam a parada de movimentos fetais ou período de 12 horas com contagem abaixo de 10 MF com maior incidência de óbito perinatal ${ }^{1,3}$. Além da mortalidade, a morbidade dos fetos com contagem de MF diminuídos também é maior, com presença de anormalidades na cardiotocografia, oligoâmnio, menores índices de Apgar e presença de mecônio no líquido amniótico ${ }^{15,16}$.

Nos últimos anos esse tema voltou a ser avaliado pela comunidade científica, estando estabelecido atualmente que a contagem de MF pode ser proposta como método de avaliação do bem-estar fetal. Autores concordam que as gestantes devem ser orientadas a respeito da contagem diária de $\mathrm{MF}^{2,5,17}$, para melhor identificação de fetos com crescimento restrito e menor incidência de neonatos com baixo escore de Apgar ${ }^{5}$. Entretanto, em revisão de literatura, não são encontrados estudos suficientes abordando a diminuição de movimentos fetais percebida pela gestante e suas consequências clínicas ${ }^{18}$.

Apesar de os métodos frequentemente utilizados para a avaliação da vitalidade fetal — cardiotocografia e perfil biofísico fetal — serem mais objetivos e precisos, necessitam de equipamentos de alta tecnologia para sua execução. Considerando-se a simplicidade e o baixo custo da contagem de MF, bem como pelo fato de prescindir de alta tecnologia e por ser acessível a qualquer mulher, sua implementação seria de grande interesse para o desenvolvimento de ações de saúde na área de atenção primária. A Society of Obstetrics and Gynaecologists of Canada ${ }^{19}$ recomenda, para gestações de alto risco, a monitorização diária dos movimentos fetais, a partir da $26^{a}$ semana. Para as gestantes saudáveis sem fatores de risco, a recomendação é de que sejam alertadas sobre a importância da movimentação fetal no terceiro trimestre e orientadas a realizar a contagem dos MF caso percebam redução. No Brasil, o Ministério da Saúde ${ }^{20}$ sugere método de contagem de MF para gestantes acompanhadas na Atenção Primária, em que, após a $34^{a}$ semana gestacional, seis MF devem ser percebidos até uma hora de observação, com repetição da contagem na hora seguinte, se necessário. Por ser de fácil aplicação, trata-se de orientação interessante para gestantes de baixo risco.

A avaliação da percepção materna dos MF em estudos anteriores demonstra concordância total de $37 \%$ entre o que é efetivamente observado ao exame ultrassonográfico ${ }^{11}$, com $30 \%$ de falsos-positivos causados por contrações uterinas, movimentos respiratórios ou sem motivos aparentes. No entanto, para movimentos grosseiros e vigorosos, a concordância é de $71 \%$ e o estudo não identifica associação com fatores como obesidade, localização da placenta ou idade materna. 
Em estudo que avalia a percepção materna dos MF, verifica-se concordância de $33 \%$ quando confrontados com a verificação por meio de dois transdutores de ultrassonografia $^{10}$. Os autores observam que $63 \%$ dos movimentos combinados são efetivamente percebidos, sem associação com a parte fetal envolvida (cabeça, tronco ou membros), duração do movimento, paridade, idade gestacional, localização da placenta ou obesidade. Para investigar a influência da duração dos MF na percepção materna, é realizada avaliação com o tocodinamômetro do aparelho de cardiotocografia e com a ultrassonografia, sendo constatada concordância de $71,2 \%$ com a ultrassonografia e de $83 \%$ para movimentos com mais de três segundos de duração ${ }^{14}$.

Não existe definição sobre o número ideal de movimentos fetais a ser utilizado em métodos de contagem, nem da sua duração para fins de vigilância do bem-estar fetal. No entanto, estudo recente demonstra que informações escritas oferecidas às gestantes sobre a importância da redução da movimentação fetal, e o estímulo ao controle desse parâmetro, promoveram melhor conduta e estão associadas a menor ocorrência de óbito fetal ${ }^{7}$.

Em conclusão, a concordância entre a percepção materna e ultrassonográfica dos MF é boa, o que permite a utilização de ferramentas de controle do bem-estar fetal baseadas nas informações maternas, como, por exemplo, o mobilograma. No Brasil, que apresenta grande demanda da população carente, a validação de um método simples, barato e disponível para todas as gestantes é de extrema importância. A boa confiabilidade da percepção materna dos MF favorece a utilização da contagem dos MF na área da atenção primária à saúde materno-fetal.

\section{Agradecimento}

À Fundação de Amparo à Pesquisa do Estado de São Paulo (FAPESP) pelas bolsas de iniciação científica concedidas aos alunos Marina Vilela Chagas Ferreira e Ibrahim Omar Abdel Latif.

\section{Referências}

1. Sadovsky E, Polishuk WZ. Fetal movements in utero: nature, assessment, prognostic value, timing of delivery. Obstet Gynecol. 1977;50(1):49-55.

2. Frøen JF. A kick from within - fetal movement counting and the cancelled progress in antenatal care. J Perinat Med. 2004; 32(1):13-24.

3. Pearson JF, Weaver JB. Fetal activity and fetal wellbeing: an evaluation. Br Med J. 1976; 1 (6021):1305-7.

4. Valentin L, Marsál K. Pregnancy outcome in women perceiving decreased fetal movement. Eur J Obstet Gynecol Reprod Biol. 1987;24(1):23-32.

5. Saastad E, Winje BA, Stray Pedersen B, Frøen JF. Fetal movement counting improved identification of fetal growth restriction and perinatal outcomes - a multi-centre, randomized, controlled trial. PLoS One. $2011 ; 6(12)$ :e28482.

6. Kuwata T, Matsubara S, Ohkusa T, Ohkuchi A, Izumi A, Watanabe $T$, et al. Establishing a reference value for the frequency of fetal movements using modified 'count to 10' method. J Obstet Gynaecol Res. 2008;34(3):318-23.

7. Tveit JV, Saastad E, Stray-Pedersen B, Børdahl PE, Flenady V, Fretts $R$, et al. Reduction of late stillbirth with the introduction of fetal movement information and guidelines - a clinical quality improvement. BMC Pregnancy Childbirth. 2009;9:32.

8. Grant A, Elbourne D, Valentin L, Alexander S. Routine formal fetal movement counting and risk of antepartum late death in normally formed singletons. Lancet. 1989;2(8659):345-9.

9. Frøen JF, Heazell AE, Tveit JV, Saastad E, Fretts RC, Flenady V. Fetal movement assessment. Semin Perinatol. 2008;32(4):243-6.

10. Hertogs K, Roberts AB, Cooper D, Griffin DR, Campbell S. Maternal perception of fetal motor activity. Br Med J. 1979;2(6199): $1183-5$.

11. Schmidt W, Cseh I, Hara K, Kubli F. Maternal perception of fetal movements and real-time ultrasound findings. J Perinat Med. $1984 ; 12(6): 313-8$.
12. Szklo M, Nieto FJ. Epidemiology: beyond the basics. 2nd ed. Sudbury: Jones and Bartlett; 2007. Chapter 8: Quality assurance and control; p. 297-349.

13. Peat AM, Stacey T, Cronin R, McCowan LM. Maternal knowledge of fetal movements in late pregnancy. Aust N Z J Obstet Gynaecol. 2012;52(5):445-9.

14. Sorokin Y, Pillay S, Dierker L, Hertz RH, Rosen MG. A comparison between maternal, tocodynamometric, and real-time ultrasonographic assessments of fetal movement. Am J Obstet Gynecol. 1981;140(4):456-60.

15. Berbey R, Manduley A, Vigil-De Gracia P. Counting fetal movements as a universal test for fetal wellbeing. Int J Gynaecol Obstet. $2001 ; 74(3): 293-5$.

16. Romero Gutiérrez G, Sánchez Cortés R, Soto Pompa V, Rodríguez Flores P. [Perinatal morbidity and mortality associated with fetal hypomotility]. Ginecol Obstet Mex. 1994;62:222-5. [Article in Spanish].

17. Erlandsson K, Lindgren H, Davidsson-Bremborg A, Rådestad I. Women's premonitions prior to the death of their baby in utero and how they deal with the feeling that their baby may be unwell. Acta Obstet Gynecol Scand. 2012;91(1):28-33.

18. Hofmeyr GJ, Novikova N. Management of reported decreased fetal movements for improving pregnancy outcomes. Cochrane Database Syst Rev. 2012;4:CD009148.

19. Liston R, Sawchuck D, Young D; Society of Obstetrics and Gynaecologists of Canada; British Columbia Perinatal Health Program. Fetal health surveillance: antepartum and intrapartum consensus guideline. J Obstet Gynaecol Can. 2007; 29(9 Suppl 4):S3-56.

20. Brasil. Ministério da Saúde. Secretaria de Atenção à Saúde. Departamento de Ações Programáticas Estratégicas. Área Técnica de Saúde da Mulher. Pré-natal e Puerpério: atenção qualificada e humanizada. Manual Técnico. Brasília (DF): Ministério da Saúde; 2005. 